\title{
Fat Harvest Using a Closed-Suction Drain
}

\author{
Kavit Amin, Roxana Zakeri, Patrick Mallucci \\ Department of Plastic and Reconstructive Surgery, Royal Free Hospital, London, UK
}

We propose a safe, simple, and novel method to harvest fat using a standard liposuction cannula and a Redivac or alternative closed-suction drain. The authors have used this technique for both 'dry' and 'wet' liposuction. This technique is both easy to perform and cost-effective whilst providing both a silent and relatively atraumatic fat harvest. The lower negative pressure compared with traditional harvesting systems likely preserves fat integrity for lipofilling. This method maximises resources already held within a hospital environment.

Keywords Liposuction / Suction / Adipose tissue

\author{
Correspondence: Kavit Amin \\ Royal free Hospital, Pond Street, \\ Hampstead, UK \\ Tel: +44-79-3195-9209 \\ Fax: +44-79-3195-9209 \\ E-mail: Kavamin@yahoo.co.uk
}

No potential conflict of interest relevant to this article was reported.

\section{INTRODUCTION}

Autologous fat transfer has increased in popularity over the past twenty years to address contour defects in all regions of the body. Autologous fat transfer has been performed since the 1890s, with the use of injectable grafts since the 1920s [1]. Adipose tissue is readily available, inexpensive, does not mount an active immune response, and can be harvested repeatedly [2]. With the expanding application of autologous fat transfer and the popularity of cosmetic lipofilling, there have been a multitude of attempts over recent years to refine techniques of fat retrieval and lipofilling, often using complex and costly equipment. Initial techniques based on blunt dissection were replaced in the 1970s with a method depending on the aspiration of fat with a vacuum suction pump. Illouz later developed 'wet' liposuction involving the use of tumescence to allow ease of aspiration of the fat [3]. This was further adapted by Klein and Lillis to enable liposuction to become commonplace as a day-case procedure performed under regional anaesthesia [2].

Suction-assisted liposuction requires the fragmentation and breakdown of fat cell walls. The fat is aspirated through a cannula into tubing and collected within a receptacle. The rate of aspiration is directly proportional to the diameter of the suction tub- ing and the vacuum pressure generated by the suction pump. Conversely, it is inversely proportional to the cannula and suction tubing length [4]. We propose a safe, simple, and novel method to harvest fat using a standard liposuction cannula and a Redivac (Atrium Medical, Hudson, NH, USA) or alternative closed suction drain (Jackson-Pratt, Cardinal health/Blake, Ethicon Inc., Somerville, NJ, USA). The authors have used this technique for both 'dry' and 'wet' liposuction.

\section{IDEA}

The suction drain is connected to standard tubing (16-gauge drain) (Fig. 1). An airtight seal between the cannula and drain tubing is vital. A $3 \mathrm{~mm}$ or $4 \mathrm{~mm}$ cannula is most effective. The vacuum must remain closed whilst the cannula is outside the cavity so the vacuum is not compromised. Whilst performing liposuction and when the cannula tip is in the body cavity, the vacuum is released and the suction from the drainage bottle aspirates fat into the bottle (Fig. 2). In the case of fat injection, the drain valve is cut at the end of the procedure (Fig. 3). A $50 \mathrm{~mL}$ syringe can be used to aspirate the fat and transfer it to smaller syringes for centrifugation. 


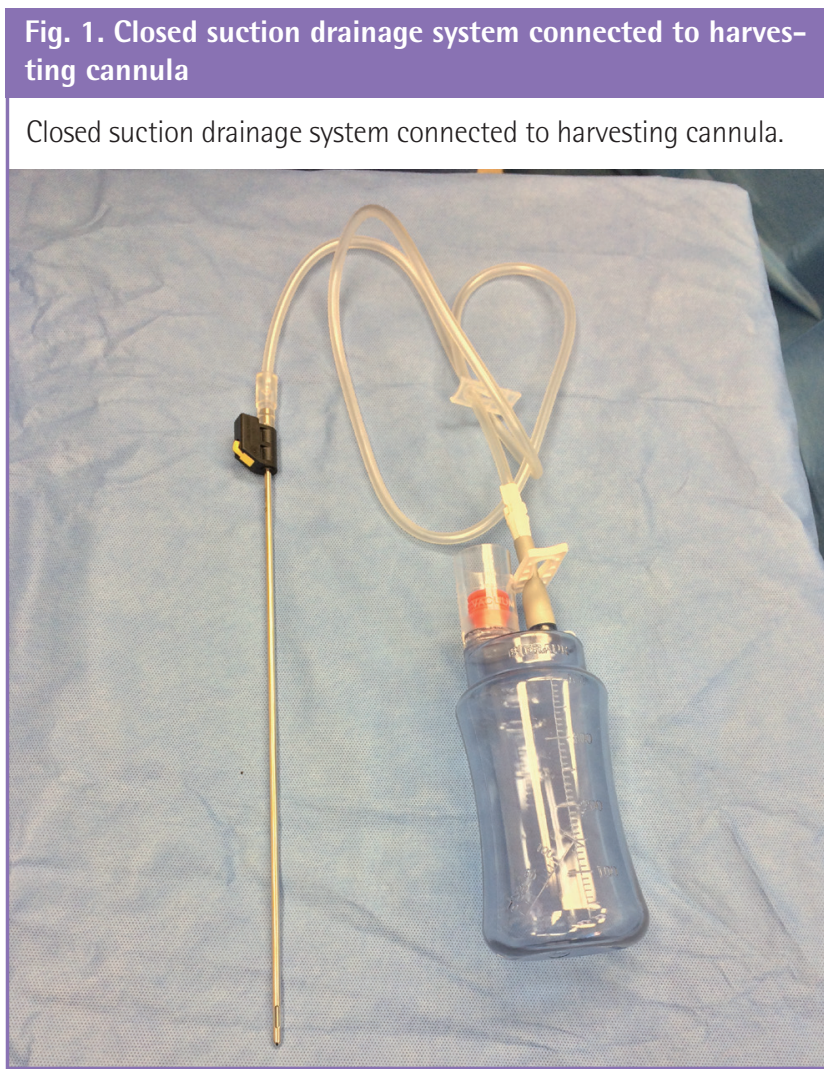

Fig. 2. Harvest of fat whilst the drainage system is closed

Harvest of fat whilst the drainage system is closed. The system is silent and easily manageable.

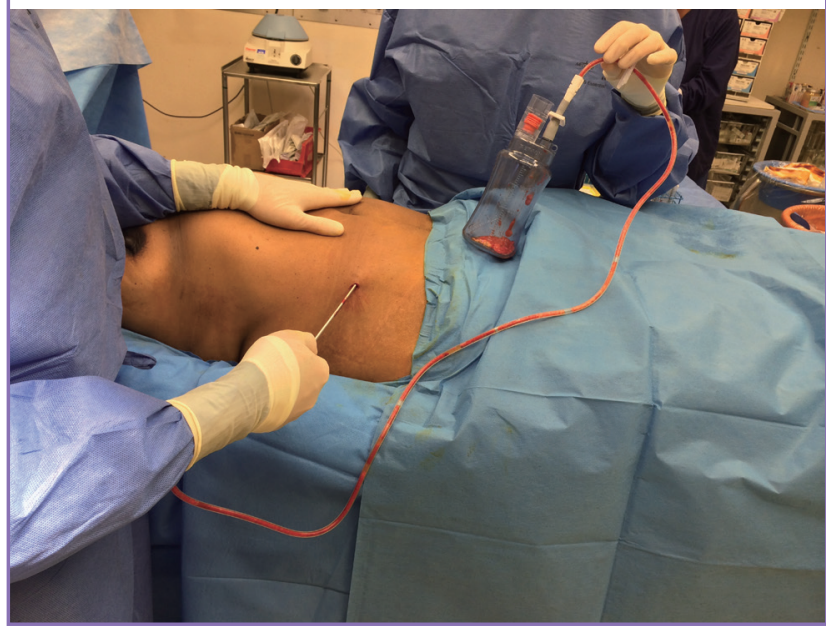

\section{DISCUSSION}

On a practical level, we have found this method to be extremely simple to perform. The Redivac/suction drain is light and attaches effortlessly to the conventional liposuction cannula. The associated tubing during aspiration from the bottle connects to a standard $50 \mathrm{~mL}$ syringe and requires minimal exertion to ex-

\section{Fig. 3. Transfer of fat to syringe if used for lipofilling}

Transfer of fat to syringe, which can then be disseminated to smaller syringes prior to centrifuge or discarded if not used for lipofilling.

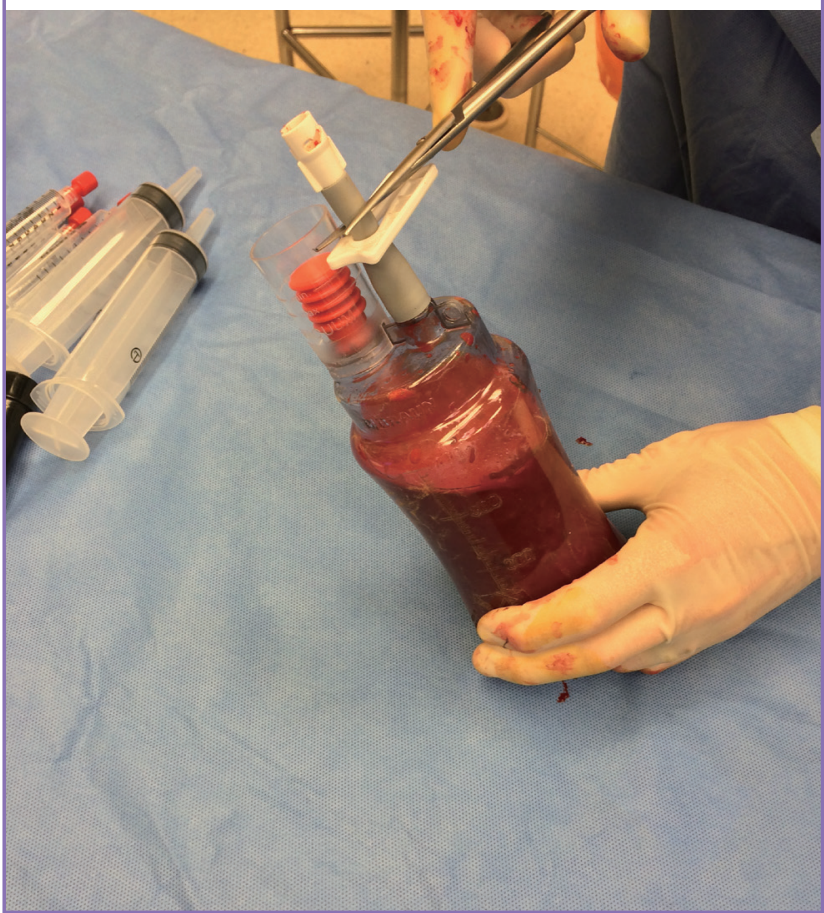

tract the aspirate. In our experience, suction pressure does not reduce significantly with increased bottle filling, even towards maximum capacity, thus negating the need for continual bottle changes. The design of the bottle with its graduated markings allows ease of recording the volumes of fat harvested. Furthermore, this technique is silent, sparing the background noise from standard suction-assisted liposuction devices. Suction drains are readily available in most theatre settings, making this technique applicable to both the office and hospital environment. Moreover they are cost-effective and disposable.

A study comparing fat harvesting with a $10 \mathrm{~mL}$ syringe and a traditional pump at suction pressures of $-350 \mathrm{~mm} \mathrm{Hg}$ and -700 $\mathrm{mm} \mathrm{Hg}$ revealed that cell yield with a pressure of $-350 \mathrm{~mm} \mathrm{Hg}$ was greater than that obtained at $-700 \mathrm{~mm} \mathrm{Hg}$ and significantly better than fat aspirated with a syringe. Therefore, it has been suggested that negative pressure is a factor that influences the quantity of stromal vascular fraction cells [5]. The suction produced by a Redivac drain is in the region of $-300 \mathrm{~mm} \mathrm{Hg}$, very similar to the $-350 \mathrm{~mm} \mathrm{Hg}$ suction described as ideal for harvesting adipose tissue on cell yield of the stromal vascular fraction [6].

Our proposed technique is ideal for small- to medium-volume liposuction, such as for facial, buttock and minor breast augmentation. The Redivac tubing readily accommodates fat lobules aspirated using 3 and $4 \mathrm{~mm}$ cannulae, with a noticeably greater 
proportion remaining intact than with the use of a machine-driven vacuum. The lower negative pressure used in this method is more likely to yield a higher quantity of viable fat graft whilst also reducing trauma to surrounding tissues, and therefore, reducing bruising and deformity postoperatively [6]. There are no added complications with this technique when compared to those of standard liposuction, in our experience [7].

Of note, this technique requires some familiarity. Common pitfalls include loss of vacuum in the bottle from withdrawing the cannula outside of the body cavity. Loss of the vacuum occurs rapidly. This can easily be addressed in the Redivac drain whereby the bottle vacuum can be reinstated by using a Yankauer suction tube whilst the clinician holds the suction device against the white plastic attachment of the vacuum bottle as described by Durai and $\mathrm{Ng}[8]$.

This useful tip is a representation of how innovation can enable maximal use of existing resources in the hospital environment.

\section{REFERENCES}

1. Kaufman MR, Miller TA, Huang C, et al. Autologous fat transfer for facial recontouring: is there science behind the art? Plast Reconstr Surg 2007;119:2287-96.

2. Botti G, Pascali M, Botti C, et al. A clinical trial in facial fat grafting: filtered and washed versus centrifuged fat. Plast Reconstr Surg 2011;127:2464-73.

3. Illouz YG. Body contouring by lipolysis: a 5-year experience with over 3000 cases. Plast Reconstr Surg 1983;72:591-7.

4. Young VL, Brandon HJ. The physics of suction-assisted lipoplasty. Aesthet Surg J 2004;24:206-10.

5. Mojallal A, Auxenfans C, Lequeux C, et al. Influence of negative pressure when harvesting adipose tissue on cell yield of the stromal-vascular fraction. Biomed Mater Eng 2008;18: 193-7.

6. Durai R, Ng PC. Perirectal abscess following procedure for prolapsed haemorrhoids successfully managed with a combination of VAC sponge and Redivac systems. Tech Coloproctol 2009; 13:307-9.

7. Sterodimas A, Boriani F, Magarakis E, et al. Thirtyfour years of liposuction: past, present and future. Eur Rev Med Pharmacol Sci 2012;16:393-406.

8. Durai R, Ng PC. Surgical vacuum drains: types, uses, and complications. AORN J 2010;91:266-71. 